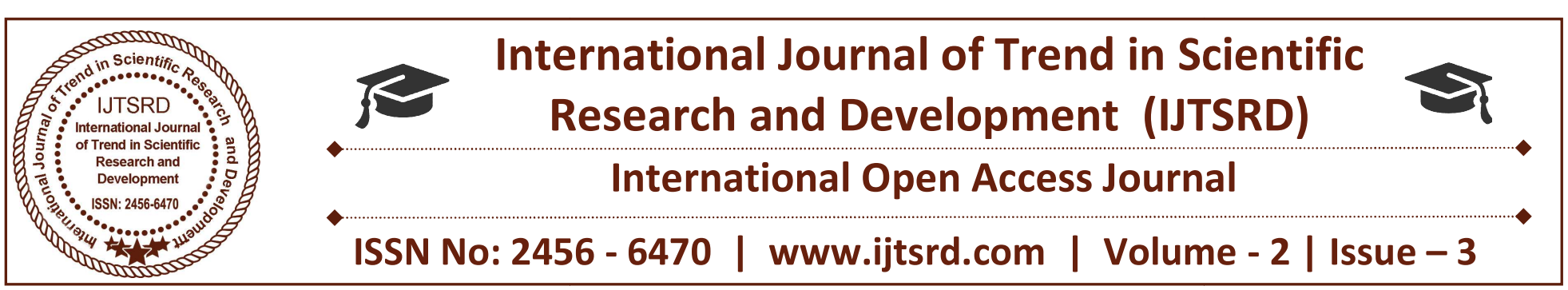

\title{
Efficiently Data Analysis and Transmission for consumer using grid computing
}

\author{
Sindhuja R \\ Research Scholar, A.V.C College of Arts and Science, \\ Mayiladuthurai, Tamil Nadu, India
}

\author{
Vasuinthira $\mathbf{N}$ \\ Asst. Professor, A.V.C College of Arts and Science, \\ Mayiladuthurai, Tamil Nadu, India
}

\section{ABSTRACT}

Research focuses on the issue of wireless big data computing in smart grid. Smart grid means electrical network architecture is purposed for generating, distributing and administering efficiently the power consumption to end-users. First investigate the consistency between the characteristics of big data and smart grid data. A propose a hybrid approach for storage planning, which consists of an outer optimization based on genetic and an inner optimization algorithm for energy scheduling. Propose a big data computing architecture for smart grid, consisting of four main levels: data sources, data transmission, data storage, and data analysis. First level, Data Source Level: data are generated from different data sources which are distributed, that is, distribution and transmission data, distributed generation data, intelligence application related data, and so on. These data are distributed in different places and managed by different companies/departments, which belong to different systems. Next, Data Storage Level: data collected from the data sources contain a lot of meaningless information, which does not need to occupy a large amount of storage space. Therefore, the data in a data center may be stored and processed. This level is suitable for the planning of smart grid with low realtime demands. Third level, Data Analysis Level: Various analytical methods and tools have been utilized to extract value in different application fields. It categorized by two division one is costumeroriented service another one is utility-companyoriented service. Final level, Data Transmission Level: This level builds a bridge among the other three levels and also enables the intra-communication within each level. Experimental results indicate that the proposed storage planning scheme significantly reduces the cost for consumers.

Keywords: smart grid, data sources, data transmission, data storage, data analysis

\section{INTRODUCTION}

The rapidly growing wave of wireless data service is pushing against the boundary of the communication network's processing power. The pervasive and exponentially increasing data traffic present imminent challenges to all the aspects of the wireless system design, such as spectrum efficiency, computing capabilities and front haul/backhaul link capacity. In this article, they discuss the challenges and opportunities in the design of scalable wireless systems to embrace such a "big data" era. On one hand, they review the state-of-the-art networking architectures and signal processing techniques adaptable for managing the "big data" traffic in wireless networks. On the other hand, instead of viewing mobile big data as a unwanted burden, they introduce methods to capitalize from the vast data traffic, for building a big data-aware wireless network with better wireless service quality and new mobile applications. They highlight several promising future research directions for wireless communications in the mobile big data area. Decades of exponential growth in commercial data services has ushered in the socalled "big data" era, to which the expansive mobile wireless network is a critical data contributor. As of 
2014, the global penetration of mobile subscribers has reached $97 \%$, producing staggeringly of mobile data worldwide. The surge of mobile data traffic in recent years is mainly attributed to the popularity of smart phones, phone cameras, mobile tablets and other smart mobile devices that support mobile broadband applications, e.g., online music, video and gaming as shown. With a compound annual growth rate of over $40 \%$, it is expected that the mobile data traffic will increase by 5 times.

In addition to the vast amount of wireless source data, modern wireless signal processing often amplifies the system's pressure from big data in pursuit of higher performance gain. For instance, MIMO antenna technologies are now extensively used to boost throughput and reliability at both mobile terminals (MTs and base stations (BSS) of high speed wireless services. This, however, also increases the system data traffic to be processed in proportion to the number of antennas in use. Moreover, the $5 \mathrm{G}$ (the fifth generation) wireless network presently under development is likely to migrate the currently hierarchical, BS-centric cellular architecture to a cloud-based layered network structure, consisting of a large number of cooperating wireless access points (APs) connected by either wire line or wireless front haul links to a big data capable processing central unit (CU). New wireless access structures, such as coordinated multipoint (networked MIMO).

On the other hand, timely and cost-efficient information processing is made possible by the fact that the vast-volume mobile data traffics are not completely chaotic and hopelessly beyond management. Rather, they often exhibit strong insightful features, such as user mobility pattern, spatial, temporal and social correlations of data contents. These special characteristics of mobile traffic present us with opportunities to harness and exploit "big data" for potential performance gains in various wireless services. To effectively utilize and exploit these characteristics, they should be identified, extracted, and efficiently stored. For instance, caching popular contents at wireless hot spots could effectively reduce the real-time traffic in the front haul links. Additionally, network control decisions, such as routing, resource allocation, and status reporting, instead of being rigidly programmed, could be made data-driven to fully capture the interplay between big data and network structure. Presently, however, these advanced data-aware features could not be efficiently implemented in current wireless systems, which are mainly designed for content delivery, instead of analyzing and making use of the data traffic.

With the increasing adoption of cloud computing, a growing number of users outsource their datasets into cloud. The datasets usually are encrypted before outsourcing to preserve the privacy. However, the common practice of encryption makes the effective utilization difficult, for example, search the given keywords in the encrypted datasets. Many schemes are proposed to make encrypted data searchable based on keywords. However, keyword-based search schemes ignore the semantic representation information of user's retrieval, and cannot completely meet with users search intention. Therefore, how to design a content-based search scheme and make semantic search more effective and context-aware is a difficult challenge. In this thesis, they proposed an innovative semantic search scheme based on the concept hierarchy and the semantic relationship between concepts in the encrypted datasets. More specifically, they scheme first indexes the documents and builds trapdoor based on the concept hierarchy. To further improve the search efficiency, utilize a tree-based index structure to organize all the document index vectors. They experiment results based on the real world datasets show the scheme is more efficient than previous scheme. They also study the threat model of they approach and prove it does not introduce any security risk.

Cloud computing is a new but increasingly mature model of enterprise IT infrastructure that provides high quality applications and services. The cloud customers can outsource their local complex data system into the cloud to avoid the overhead of management and local-storage. However, the security of outsourced data cannot be guaranteed, as the Cloud Service Provider (CSP) possesses whole control of the data. So, it is necessary to encrypt data before outsourcing them into cloud to protect the privacy of sensitive data. However, encryption for outsourced data can protect privacy against unauthorized behaviors; it also makes effective data utilization, such as search over encrypted data, a very difficult issue. In recent years, many researchers have proposed a series of efficient search schemes over encrypted cloud data. The general process of search scheme is divided into five steps: extracting document features, constructing a searchable index, generating search trapdoor, searching the index based on the trapdoor and returning the search results. These search 
schemes provide different query capabilities, including single keyword search, multi-keyword search, fuzzy keyword search, similarity search, and so on. However, all the existing searchable encryption schemes, which consider keywords as the document feature, do not take the semantic relations between words into consideration, both in the steps of extracting document features and generating search trapdoor. As they all know, the semantic relations between words are diverse, such as synonymy, domain correlation. Considering the potentially huge amount of outsourced data documents in the cloud, the search accuracy and search efficiency are influenced negatively if the semantic relations between words are not handled well.

In this paragraph, they make a detailed description of existing problems of the available searchable schemes. Firstly, in the stage of extracting document features, the data owner computes the weight of each word in a document and then selects $t$ words with top$\mathrm{t}$ weights as the feature of the document. In the process shown above, every two words with different spelling are assumed uncorrelated, which is unreasonable. For example, three words "network", "net", "web" are different in the perspective of spelling, but they are semantically related, that is they are synonym. It is obvious that the weight of word is influenced if semantic relations between words are ignored and the accuracy of the document features is influenced consequently. Secondly, in the stage of generating search trapdoor, the trapdoor is generated only based on the search keywords inputted by the data user, which is inflexible, because it is impossible to extend the search keywords when the data user cannot well express his search intention. In this case, it is likely that a returned document is not needed for the data user or the really needed documents are not returned. So, it is important to understand the real search intention of the data user to avoid returning unnecessary documents to improve search efficiency, as the size of the document set outsourced into the cloud server is potentially huge. Thirdly, a search request usually focuses on a theme, and some search words can be considered to be the attribute of the theme, for example, "birthday" is an attribute of a people. In existing search schemes, an attribute value is usually treated as a keyword which ignores the relationship with the mean results in larger keyword dictionary, and then negatively influences the search accuracy and efficiency. Therefore, it is a very important and challenging task to implement semantic search over encrypted data.

Distributed devices in smart grid systems are decentralized and connect to the power grid through different types of equipment transmit, which will produce numerous energy losses when power flows from one bus to another. One of the most efficient approaches to reduce energy losses is to integrate distributed generations (DGs, mostly renewable energy sources). However, the uncertainty of DG may cause instability issues. Additionally, due to the similar consumption habits of customers, the peak load period of power consumption may cause congestion in the power grid and affect the energy delivery. Energy management with DG regulation is considered to be one of the most efficient solutions for solving these instability issues. In this thesis, they consider a power system with both distributed generators and customers, and propose a Distributed Locational Marginal Pricing (DLMP)-based unified Energy Management System (UEMS) model which, unlike previous works, considers both increasing profit benefits for DGs and increasing stability of the distributed power system (DPS).

The model contains two parts: a game theory based loss reduction allocation (LRA) and a load feedback control (LFC) with price elasticity. In the former component, they develop an iterative loss reduction method using DLMP to remunerate DGs for their participation in energy loss reduction. By using iterative LRA to calculate energy loss reduction, the model accurately rewards DG contribution and offers a fair, competitive market. Furthermore, the overall profit of all DGs is maximized by utilizing game theory to calculate an optimal LRA scheme for calculating the distributed loss of every DG in each time slot. In the latter component of the model, they propose an LFC sub-model with price elasticity, where a DLMP feedback signal is calculated by customer demand to regulate peak-load value. In UEMS, LFC first determines the DLMP signal of a customer bus by a time-shift load optimization algorithm based on the changes of customer demand which is fed back to the DLMP of the customer bus at the next slot-time, allowing for peak-load regulation via price elasticity. Results based on the IEEE 37-bus feeder system show that the proposed EMS model can increase DG benefits and improve system stability.

The smart grid is seen as the best approach in modernization of electric power systems, and the DPS 
is one of the most significant parts. The decentralized devices in the DPS will produce high system loss while power flow transmit from one bus to another through the branch or some power devices such as substation, and the similar power-consuming habit of customer, which is the main reason to cause peak-load period of power consumption, may aggravate the loss reduction problem. Thus, energy management system should be designed to dynamically adapt to DPS by controlling and regulating distributed devices to make power systems more effective and reliable. As for the application of energy management, using load forecasting to regulate energy distribution in microgrid is very hot in recent research. The authors in presented an electric load forecast architectural model to integrate distributed renewable sources. This is to balance power generation and demand in order to offer a good service at a competitive price. Integration of DGs in DPS could greatly enhance the competitiveness power of distributed companies in a competitive environment, and provide benefits for energy loss reduction and improve stability in the grid.

In order to perform some types of complex tasks, the principles of crowd sourcing have been applied in constructing a mobile crowd sourcing network with a huge amount of human involvement and powerful mobile devices. In addition, through the social Internet of Things (SIOT) paradigm, the capability of humans and devices to discover and exploit objects with their services is augmented. Thus, SIOT has become a promising network for service discovery and composition. By introducing SIOT, they can expand the traditional mobile crowd sourcing network and achieve broader crowd sourcing application. However, winner selection and payment determination by evaluating the reliability of crowd sourcing participants. Finally, some challenges in trustworthy crowd sourcing are discussed.

With the rapid development in computing and communication technologies, more and more objects are being equipped with processors, sensors, memories, and wireless communication modules that enable object-to-object communication, forming the Internet of Things (IOT). It is demonstrated that many issues need to be considered in order to implement the effectiveness and reliability of IOT, such as the sensing world, transmitting data, and building applications based on relevant services. Similar to social networks for human beings, the social IOT (SIOT), introduces social relationships among things and creates trustworthiness, leveraging the interaction among things. With the popularity of smart phones and other intelligent devices, objects can easily communicate with each other without the restriction of time and place. There is a significant trend that takes full advantage of mobile devices and applications to support crowd sourcing to solve a diversity of issues involving real-time data acquisition, processing, and collaborations among a large number of participants in mobile environments.

Obviously, crowd sourcing can bring not only great social and economic benefits, but also real changes in the daily lives. In traditional mobile crowd sourcing networks, the crowd sourcing tasks are received by participants who possess mobile devices with computing power or sensing capacity. To amplify the function of mobile crowd sourcing networks, they introduced the SIOT paradigm into crowd sourcing, in which objects as sensing entities can discover services and resources through forming social relationships. This provides a crowd sourcing solution in which mobile devices transform its role for participants into sensing entities.

\section{Efficiently Data Analysis and Transmission for consumer using grid computing:}

\section{Approach:}

The hybrid approach combines the semantic, corpus, ontology and relational based approaches. A novel hybrid approach extracts semantic knowledge from the structural representation of Word net and the statistic information on the Internet. Internet based semantic knowledge estimates the semantic similarity between the two concepts in Word net. A useful measure called Normalized Google Distance (NGD) computes the semantic distance between the adjacent concepts, along the shortest path in Word Net using Internet semantic knowledge. It is one of the best approaches due to the deployment of Internet knowledge in Word Net based semantic relatedness measure. Between sentences and documents. This work measures the similarity between the documents using both ontology-based similarity model and counts based similarity model. Shows the components used in this methodology. Initially, the proposed system represents the input documents as a bag of words, and it avoids the repeated terms. The preprocessing step removes stop words from the representation. The important keywords in the documents are identified using the term frequency 
mechanism. Term frequency determines the similarity measure estimates the similarity between frequently occurring words in a document as the documents by dividing the number of commonly keywords. The derived keywords are given to the related keywords by the total number of total ontology to obtain related keywords. The related keywords.

keywords are given to the Jaccard similarity coefficient to decide the documents are similar or not. The Jaccard similarity coefficient is the count based

Architecture view:

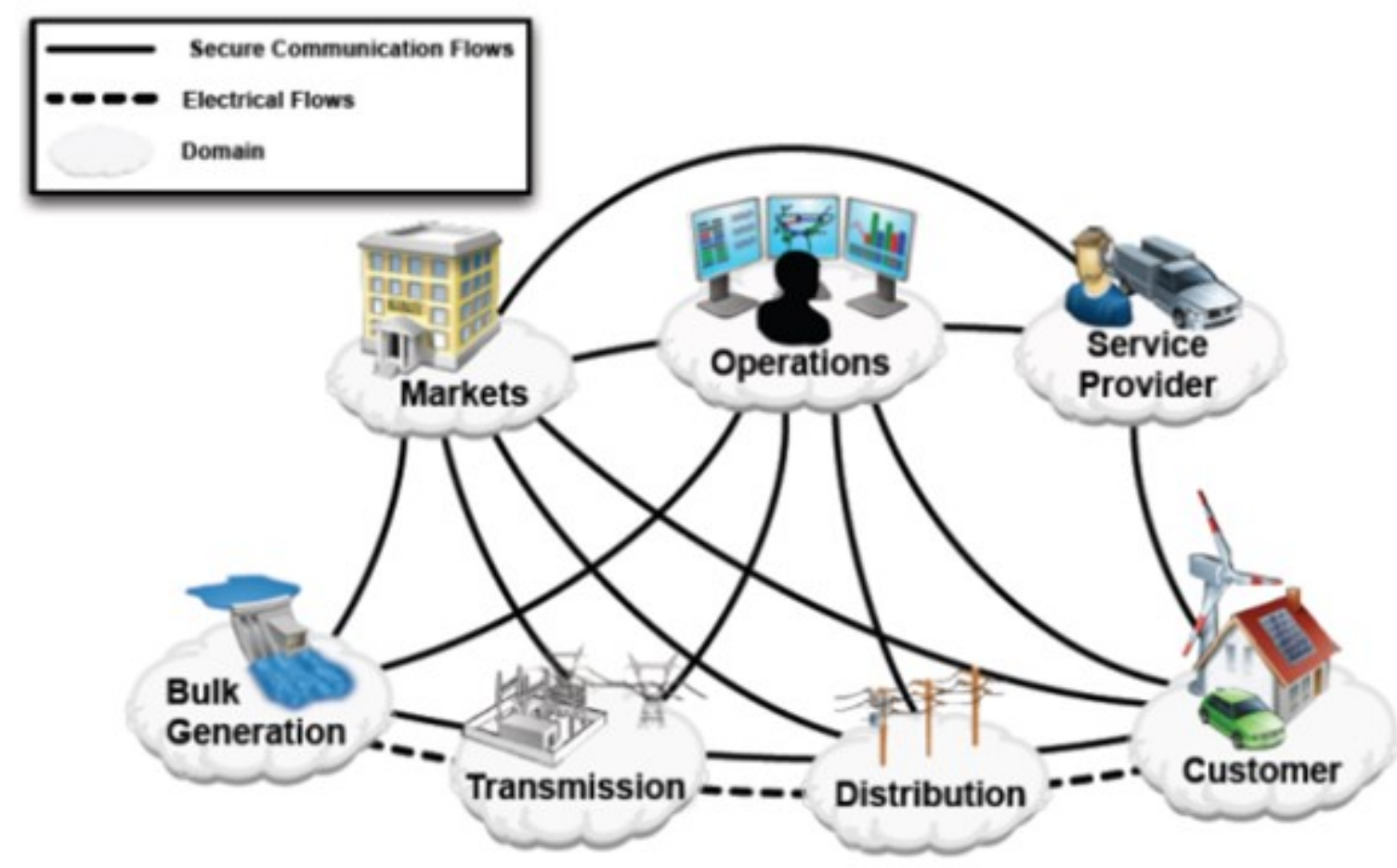

Algorithm:

1: Choose an initial random population of individuals

2: Evaluate the fitness of the individuals

3: repeat

4: $\quad$ Select the best individuals to be used by the genetic operators

5: Generate new individuals using crossover and mutation

6: Evaluate the fitness of the new individuals

7: $\quad$ Replace the worst individuals of the population by the best new individuals

8: until some stop criteria 


\section{Problem definition:}

The cloud services technology as the basis for communication and management mechanism in order to provide a strong support for power monitoring and early-warning system. With the fast development of smart grid, higher storage capacity and stronger processing ability of power system platform are needed .A smart grid can be conceptualized as a cyber-physical system that connects physical electricity systems and cyber-infrastructure, with the integration of the Internet. This service can communicate with the consumer appliances and also provide the backbone for utilities to assimilate content and control operations. With the presence of online connectivity, smart grids have greater exposure to cyber-attacks that can potentially disrupt the power supply. One of the important issues is power theft by consumers. This can be done by hacking a smart meter or changing its communication channel to change the reported electricity usage. Additionally, data manipulation is also one of the most security concerns in the smart grid. To overcome these issues in they work.

\section{Existing System:}

Existing approach of Cloud-Based Demand Response (CDR). It is fast response times in large scale deployment. In this architecture, the master/slave demand response model is proposed, in which the smart meters and the home EMS act as slaves, and the utility acts as the master. In such a scenario, the CDR leverages data-centric communication, publisher/subscriber and topic-based group management, instead of IP-centric communication. Two cloud-based demand response models are proposed as follows: (a) data-centric communication and (b) topic-based group communication. Secure, scalable and reliable demand response can be achieved by using the CDR approach. However, the demand-response model discussed in and with the implementation of private cloud for a small-sized network. Some of the overhead problems are the implementation cost, and the selection of appropriate strategy. Even for a small-sized network, all the features of cloud computing platform should be supported in order to have reliable, and secure electricity distribution in a smart grid, and, thus, implementation cost of cloud applications is higher for a small-sized network. So, there is a need for developing such a demand-response model using cloud-based applications.

\section{Demerits:}

$>$ It does not facilitate both the large and smallscale network.

$>$ Implementation cost is high.

$>$ It does not satisfy the consumer requirement.

$>$ It does not flexible for on demand request.

\section{Proposed System:}

Propose a hybrid approach for storage planning, which consists of an outer optimization based on Genetic and an inner optimization algorithm for energy scheduling. Propose a big data computing architecture for smart grid, consisting of four main levels: data sources, data transmission, data storage, and data analysis. First level, Data Source Level: data are generated from different data sources which are distributed, that is, distribution and transmission data, distributed generation data, intelligence application related data, and so on. These data are distributed in different places and managed by different companies/departments, which belong to different systems. Next, Data Storage Level: data collected from the data sources contain a lot of meaningless information, which does not need to occupy a large amount of storage space. Therefore, the data in a data center may be stored and processed. This level is suitable for the planning of smart grid with low realtime demands. Third level, Data Analysis Level: Various analytical methods and tools have been utilized to extract value in different application fields. It categorized by two divisions one is costumeroriented service another one is utility-companyoriented service. Final level, Data Transmission Level: This level builds a bridge among the other three levels and also enables the intra-communication within each level.

\section{Merits:}

$>$ Easy to Analysis the data.

$>$ High performance.

$>$ Reduce the time.

$>$ Accurate result.

\section{Experimental Result:}

\section{Maximum Throughput:}

In this experiment throughput. LDP gives the $45 \%$ of the throughput. FTP gives the $55 \%$ of the throughput. EBT gives the $63 \%$ of the throughput. GA gives the $78 \%$ of the throughput. 


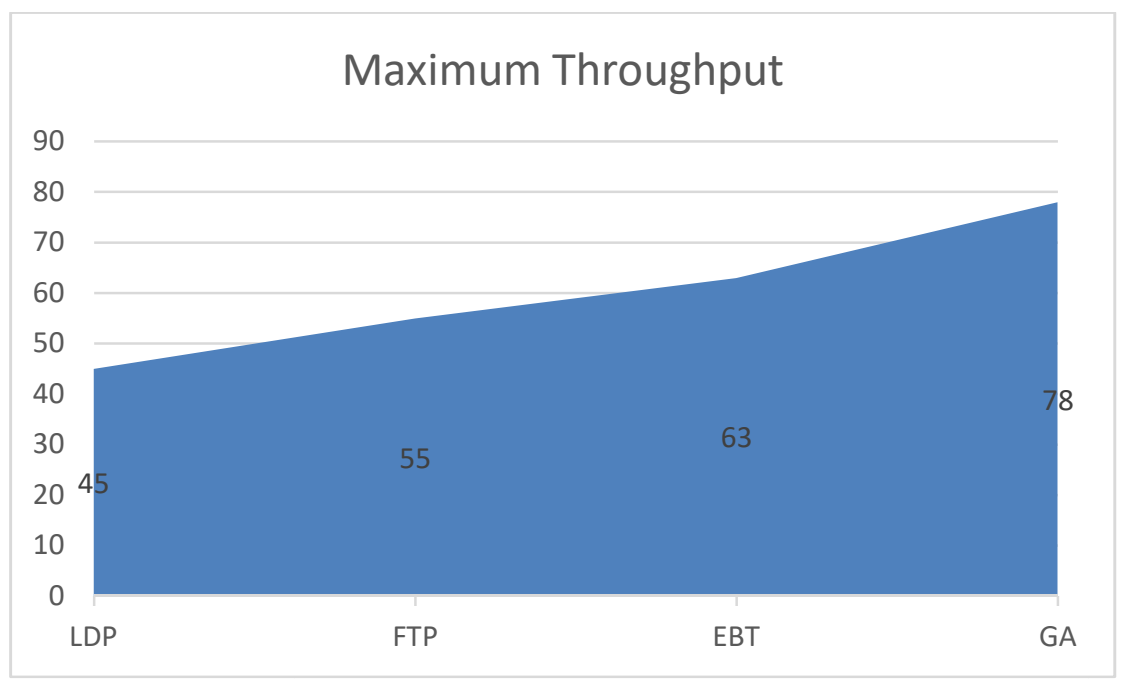

\section{COST:}

In this experiment cost. LDP gives the $73 \%$ of the cost. FTP gives the $60 \%$ of the cost. EBT gives the $50 \%$ of the cost. GA gives the $35 \%$ of the cost.

\section{High Efficiency:}

In this experiment efficiency. LDP gives the $60 \%$ of the efficiency. FTP gives the $40 \%$ of the efficiency. EBT gives the $55 \%$ of the efficiency. GA gives the $83 \%$ of the efficiency.

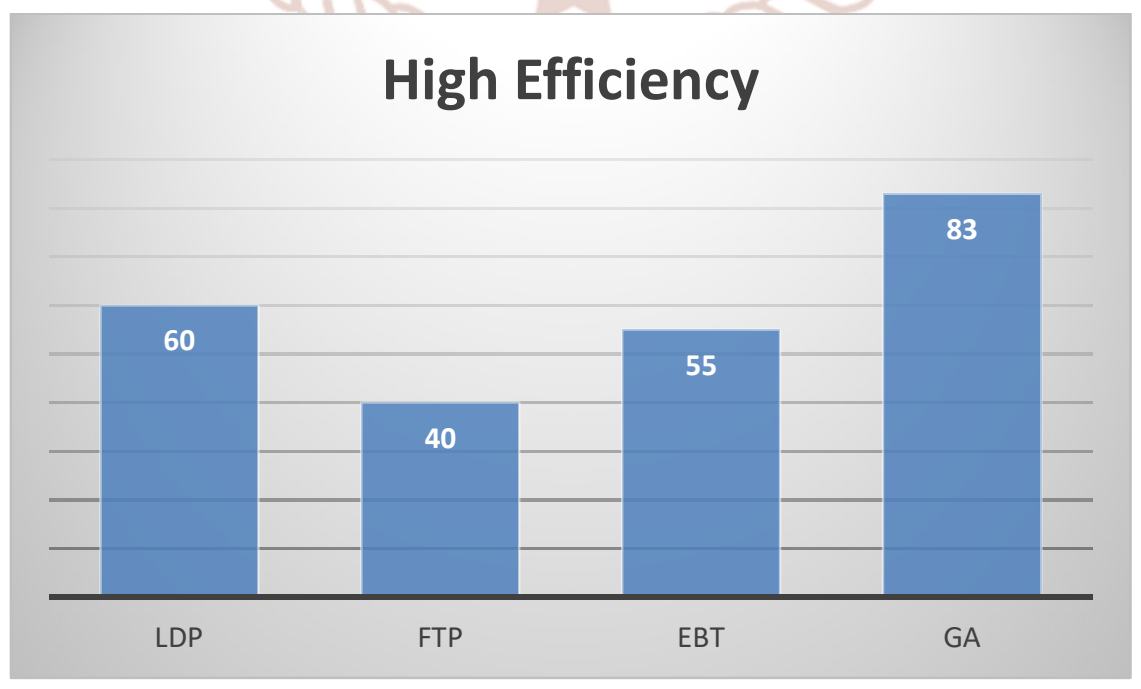




\section{Conclusion:}

In this thesis, they provided an overview of integrating cloud computing in the smart grid architecture, in order to have reliable, efficient and secure energy distribution. Different aspects of smart grid energy management, information management, and security are discussed. In this article, thesis have discussed the characteristics of smart grid big data and proposed a big data computing architecture for smart grid analytics. Then the hierarchical big-data-aware wireless communication architecture for smart grid has been proposed, and enabling technologies are introduced.

\section{Future Work:}

In future, cloud computing even gives better security capability than the conventional, pure IP-based security mechanisms. Thus, the integration of cloud computing in smart grid is envisioned to be useful for evolving the smart grid architecture further in terms of considerations such as monitoring cost, computing, data management, power management, and security.

\section{Reference:}

1) SoumenChakrabarti Ganesh Ramakrishnan, "Data-based Research at IIT Bombay", 2017.

2) Rong Yu,ShengliXie, "Cognitive-Radio-Based Hierarchical Communications Infrastructure for Smart Grid”, 2017.

3) Hervais Simo, "Big Data: Opportunities and Privacy Challenges", 2016.
4) Zhanyu Ma, JiyangXie, "The Role of Data Analysis in the Development of Intelligent Energy Networks", 2015.

5) Kyri Baker, Gabriela Hug, “Optimal Integration of Intermittent Energy Sources Using Distributed Multi-Step Optimization”, 2015.

6) Werner Vogels, "Big Data: Challenges and Opportunities”, 2014.

7) Khaled Shuaib., EzedinBarka, "Cognitive Radio for Smart Grid with Security Considerations", 2014.

8) Khaled Shuaib., EzedinBarka, "Cognitive Radio for Smart Grid with Security Considerations", 2014.

9) Aneri M. Desai, Rutvij H. Jhaveri, "A Review on Applications of Ambient Assisted Living", 2013.

10) Diksha M., Raghavendra T. S, “A Survey on Big Data Energy Based on Smart Grid”, 2014.

11) Suzhi Bi, Rui Zhang, Zhi Ding, "Wireless Communications in the Era of Big Data", 2013.

12) IBM Corp. Software Group, "IBM Big Data Overview forEnergy and Utilities"; http://www01.ibm.com/software/tivoli/solution/industry/ener gy-utilities, 2012.

13) R. Yu et al., "Hybrid Spectrum Access in Cognitive-Radio-Based Smart-Grid Communications Systems," IEEE Sys. J.,vol. 8, no. 2, 2014, pp. 577-87. 